\title{
TESTE DO PEZINHO E O PAPEL DA ENFERMAGEM: UMA REFLEXÃO
}

\section{THE BLOOD DROP SCREENING (GUTHRIE) TEST AND THE ROLE OF NURSES: A REFLECTION}

\section{GUTHRIE PRUEBA Y EL PAPEL DE LA ENFERMERÍA: UNA REFLEXIÓN \\ Alessandra Bernadete Trovó de Marqui ${ }^{1}$}

\section{RESUMO}

OBJETIVO: refletir e re(pensar) a atuação da Enfermagem no Teste do Pezinho. METODOLOGIA: trata-se de um estudo descritivo-reflexivo com fundamentação teórica sobre a prática de enfermagem em Triagem Neonatal. RESULTADOS: a literatura mostra: 1) fragilidades na atuação desses profissionais em Triagem Neonatal e 2) entendimento limitado das mães sobre o teste, que pode ser reflexo da atuação inadequada da Equipe de Enfermagem na Educação em Saúde. CONCLUSÕES: para que a Enfermagem atue na promoção da saúde e prevenção de doenças, é indispensável que o profissional reflita sobre seu papel social e busque continuamente por capacitação, pois essa lhes proporcionará uma melhoria na qualidade da assistência prestada à mãe e recém-nascido.

DESCRITORES: Enfermagem; Triagem Neonatal; Saúde da Criança; Educação em Saúde

\begin{abstract}
OBJECTIVE: to evaluate and possibly propose a repositioning of the role of nurses in the Blood Drop Screening (Guthrie) Test (GT). METHODOLOGY: this is a descriptivereflexive study with theoretical grounds of the nursing practice in Neonatal Screening. RESULTS: the literature shows: 1) weak points in the performance of these professionals in neonatal screening and 2) limited understanding of mothers regarding the test, which may reflect the inadequate performance of the Nursing Team in Health Education. CONCLUSIONS: if nurses are to act effectively in health promotion and disease prevention, it is essential that they are aware of their social role and willing to continuously improve their knowledge and skills. This will enable them to provide better care both to the mother and the newborn.
\end{abstract}

DESCRIPTORS: Nursing; Neonatal Screening; Child Health; Health Education

\section{RESUMEN}

OBJETIVO: reflexionar y volver a (pensar) el papel de la enfermería en la prueba de Guthrie. METODOLOGÍA: se trata de un estudio descriptivo-reflexiva con el marco teórico de la práctica de enfermería en el Tamizaje Neonatal. RESULTADOS: la literatura muestra: 1) debilidades en el desempeño de estos profesionales en Tamizaje Neonatal y 2) la comprensión limitada de las madres en la prueba, lo que puede reflejar el comportamiento inadecuado del equipo de enfermería en la Educación en Salud. CONCLUSIONES: para la actuación de enfermería en la promoción de salud y prevención de enfermedades, es esencial que los profesionales de la salud creen que su rol social y la búsqueda continua de la formación, ya que esto les dará una mejora en la calidad de la atención proporcionada madre y el recién nacido.

DESCRIPTORES: Enfermería; Tamizaje Neonatal; Salud del Niño; Educación en Salud

\footnotetext{
1 Associate professor of the Genetics Discipline at Universidade Federal do Triângulo Mineiro/UFTM, Uberaba/MG.
} 


\section{INTRODUÇÃO}

\section{Teste do Pezinho e Triagem Neonatal}

O Teste do Pezinho (TP) é um exame laboratorial que faz parte da Triagem Neonatal (TN), juntamente com os testes da orelhinha, dos olhinhos, da linguinha e do coraçãozinho e são importantes para diagnosticar distúrbios que possam prejudicar o desenvolvimento do recém-nascido $(\mathrm{RN})$. O TP detecta doenças metabólicas, genéticas e/ou infecciosas, antes que os sintomas tornemse evidentes, facilitando a inclusão do RN em tratamento específico a fim de diminuir ou eliminar sequelas associadas a cada doença, permitindo uma melhor qualidade de vida à criança. ${ }^{1-3}$

A TN é o maior programa de saúde pública preventiva no mundo. No Brasil, trata-se da maior iniciativa do Sistema Único de Saúde (SUS) na área de Genética. Foi implantada em nosso país em 1976 pelo Dr. Benjamin José Schmidt, médico pediatra da APAE/SP (Associação de Pais e Amigos dos Excepcionais) que implementou o diagnóstico para Fenilcetonúria. Em 1986 foi incluído a triagem para Hipotireoidismo Congênito. Na década de 90, o rastreamento para essas duas patologias ficou conhecido como TP porque as amostras de sangue são obtidas do calcanhar do $\mathrm{RN}$, por ser essa uma região altamente vascularizada e praticamente indolor para o bebê. Em
2001, o governo federal implantou por meio da Portaria no. 822 de 06 de junho o Programa Nacional de Triagem Neonatal (PNTN), que estabelece que toda criança nascida em território nacional deve ser submetida ao TP, que é gratuito e obrigatório. ${ }^{4}$ A implantação da TN no Brasil foi realizada em fases (I, II e III), de acordo com o nível de organização e de cobertura de cada estado, e portanto, os distúrbios investigados variam de estado para estado. Em 2012 houve a expansão para a fase IV e a inclusão de mais duas doenças a serem rastreadas. ${ }^{5}$ Atualmente, as doenças diagnosticadas pelo TP são seis e incluem Fenilcetonúria, Hipotireoidismo Congênito, Anemia Falciforme e outras Hemoglobinopatias, Fibrose Cística, Hiperplasia adrenal congênita e Deficiência de Biotinidase. Tais doenças são assintomáticas no período neonatal e, portanto, não despertam a atenção médica, sendo, portanto, imprescindível o diagnóstico precoce pelo TP. Assim, o PNTN tem como principal meta a prevenção e redução da morbimortalidade provocada pelas patologias triadas e em caso positivo, o tratamento precoce antes do surgimento de sequelas irreversíveis como a deficiência intelectual. As patologias detectadas pelo TP são incuráveis, porém, apresentam um bom prognóstico se diagnosticadas e tratadas desde o período neonatal. ${ }^{1-3}$ 
Nesse contexto, o presente trabalho teórico tem caráter descritivo-reflexivo e visa re(pensar) a atuação da Enfermagem no TP. Para tanto, foram consultados periódicos publicados sobre essa temática até o momento e os resultados apresentados por esses estudos foram discutidos criticamente e constituíram a fundamentação teórica dessa pesquisa.

\section{Teste do Pezinho e Enfermagem}

A Enfermagem, Obstetrícia e Pediatria são fundamentais para sucesso da $\mathrm{TN}$ pois esses profissionais atuam no atendimento da gestante, parturiente, RN e puérpera e devem ter conhecimento dos distúrbios metabólicos, bem como da importância do diagnóstico precoce das enfermidades pesquisadas no PNTN. Os pais precisam saber da existência da $\mathrm{TN}$ e ser orientados previamente sobre: os benefícios da detecção precoce das doenças a serem triadas e quais são elas; os riscos existentes para o $\mathrm{RN}$ que não é submetido ao teste; a idade adequada para sua realização; a necessidade de exames confirmatórios posteriores para os que foram positivos; a possibilidade de falsopositivos; o processo de acompanhamento e recebimento dos resultados. ${ }^{3}$ Dentre esses profissionais de saúde, o enfermeiro é quem mais interage com a clientela alvo: a mãe e o RN. Desde o pré-natal, nas Unidades Básicas de Saúde, o enfermeiro deve informar e orientar a gestante que quando o seu bebê nascer ele fará um exame, chamado TP, que pode ser realizado no sistema público ou particular de saúde. $\mathrm{Na}$ maternidade/hospital, novamente a Enfermagem deverá reforçar tais informações aos pais, especialmente para mães primigestas. ${ }^{6}$ A importância do exame, o esclarecimento sobre a finalidade da coleta e o procedimento em si e a necessidade de buscar o resultado do exame são orientações que, durante a assistência, permitem à mãe a sensação de segurança, oferecendo a ela saberes que consolidam a responsabilidade no sentido de promover o bem-estar e apoio à saúde de seu filho. ${ }^{7}$

A técnica correta de coleta das amostras de sangue para o TP é um procedimento de Enfermagem. Esse profissional deve preencher corretamente a ficha de coleta para facilitar a localização da criança caso seja necessário, como por exemplo, repetição ou resultado alterado no exame. Com o auxílio de uma lanceta são obtidas algumas gotas de sangue de uma das laterais do calcanhar do bebê e estas são depositadas em papel filtro. O período para realizar o exame deve ser do $3^{\circ}$ ao $7^{\circ}$ dia de vida do $\mathrm{RN}$, preferencialmente no $5^{\circ}$ dia, pois o mesmo já esteve em contado com o leite materno, que é fonte de alimentação protéica. Deste modo, deve ser realizado com no mínimo 
48 horas de vida do $\mathrm{RN}$ e nunca superior a 30 dias. É importante que o profissional de saúde informe aos pais que caso o resultado dê positivo deve-se fazer o teste diagnóstico, pois a TN não é confirmatória assim pode haver falsos - positivos e falsos - negativos. $^{1,2}$

Apesar da coleta do TP ser um procedimento de competência do enfermeiro, estudos têm demonstrado falha na atuação desses profissionais. ${ }^{6,8,9} \mathrm{Um}$ deles mostrou que das 222.366 amostras de sangue coletadas em papel filtro para o TP no estado do Paraná, 2.787 (1,25\%) delas exibiram erros na coleta. ${ }^{6,8}$ Os principais motivos foram: 1198 exames por sangue insuficientes, 500 exames por sangue envelhecido e 490 por sangue ressecado. Esses dados chamam a atenção por mostrarem uma falta de conhecimento técnico científico e desvalorização quanto à importância do exame pelos profissionais de enfermagem. ${ }^{6,8}$ Outro estudo, conduzido em um Hospital Universitário do Paraná, mostrou um número elevado de $2^{\mathrm{a}}, 3^{\mathrm{a}}$ e $4^{\mathrm{a}}$ coletas de amostras do TP, totalizando $\mathrm{n}=110(11,3 \%)$. Esse número de recoleta sem motivo registrado evidenciou falhas durante o exame seja na coleta, armazenamento ou registro. ${ }^{9}$ As recoletas de material para o TP agridem física e emocionalmente o bebê e sua família e evidenciam a necessidade de um treinamento específico para o profissional de enfermagem. ${ }^{9}$ Ainda, uma má coleta de amostra para o TP, ou seja, quando uma amostra de sangue não é coletada com a qualidade exigida, a mãe é reconvocada para repetir o exame do seu bebê o mais urgente possível e isso provoca, além de ansiedade, insegurança, desconforto e estresse à família e ao bebê, um significativo atraso no diagnóstico e início tardio do tratamento nos casos positivos para alguma das doenças pesquisadas, principalmente se houver dificuldade em localizar essa mãe e seu bebê. ${ }^{6}$

Ainda, outros estudos têm demonstrado algumas fragilidades quanto ao conhecimento da Equipe de Enfermagem sobre o TP (Quadro 1). ${ }^{10-12}$

Quadro 1 - Sumário dos principais resultados sobre conhecimento da Enfermagem sobre TP.

\begin{tabular}{|c|c|c|c|}
\hline Referência & Objetivo & Casuística & Principais resultados \\
\hline $\begin{array}{l}\text { Benincasa et al. } \\
10\end{array}$ & $\begin{array}{l}\text { Identificar a percepção teórica } \\
\text { da equipe de enfermagem } \\
\text { sobre TN em Unidade de } \\
\text { Terapia Intensiva Neonatal de } \\
\text { um hospital público do } \\
\text { interior paulista. }\end{array}$ & $\begin{array}{lr}21 \text { profissionais } & \text { de } \\
\text { enfermagem } & (4 \\
\text { enfermeiros, } & 14 \\
\text { técnicos e } & 3 \\
\text { auxiliares } & \mathrm{de} \\
\text { enfermagem) } & \end{array}$ & $\begin{array}{l}\text { - } 19 \text { profissionais da equipe não } \\
\text { associaram o termo TN com TP; } \\
\text { - Nenhum dos } 21 \text { entrevistados } \\
\text { respondeu de forma correta o } \\
\text { período preconizado para coleta; } \\
\text { - Nenhum dos } 21 \text { citou a real } \\
\text { importância da TN que é a } \\
\text { prevenção de complicações que } \\
\text { as patologias triadas ocasionam }\end{array}$ \\
\hline
\end{tabular}




\begin{tabular}{|c|c|c|c|}
\hline & & & $\begin{array}{l}\text { à vida da criança se, contudo, } \\
\text { não diagnosticadas e tratadas } \\
\text { precocemente; } \\
\text { - Nome das patologias triadas } \\
\text { (SP: três patologias): } \\
6 \text { mencionaram uma } \\
\text { patologia } \\
3 \text { citaram duas } \\
8 \text { não mencionaram nenhuma } \\
\text { das três patologias } \\
4 \text { responderam essa questão } \\
\text { corretamente }\end{array}$ \\
\hline Acosta et al.11 & $\begin{array}{l}\text { Conhecer as orientações } \\
\text { acerca da TN, compartilhadas } \\
\text { pelos enfermeiros com } \\
\text { pais/mães }\end{array}$ & $\begin{array}{l}13 \text { enfermeiras que } \\
\text { atuam nas Unidades } \\
\text { Básicas de Saúde da } \\
\text { Família (UBSF) }\end{array}$ & $\begin{array}{l}\text { - Apenas três enfermeiras } \\
\text { utilizam estratégias de ensino- } \\
\text { aprendizagem para facilitar o } \\
\text { entendimento dos familiares } \\
\text { sobre TP. As orientações foram } \\
\text { dadas a partir do conhecimento } \\
\text { prévio e da capacidade de } \\
\text { absorção do cuidador; } \\
\text { - Apenas uma enfermeira relatou } \\
\text { a importância da abordagem do } \\
\text { tema antes mesmo do } \\
\text { nascimento do bebê, ou seja, } \\
\text { durante o pré-natal; } \\
\text { - Equívoco em relação ao tempo } \\
\text { indicado para coleta do exame. }\end{array}$ \\
\hline Strefling et al.12 & $\begin{array}{l}\text { Descrever o conhecimento das } \\
\text { enfermeiras sobre } \\
\text { a TN e sua operacionalização }\end{array}$ & $\begin{array}{l}13 \text { enfermeiras que } \\
\text { atuam nas UBSF }\end{array}$ & $\begin{array}{l}\text { - Necessidade de maior } \\
\text { aprofundamento sobre o tema; } \\
\text { - Fragilidades quanto à } \\
\text { orientação do período } \\
\text { apropriado para coleta do } \\
\text { exame; } \\
\text { - Relato de procedimentos } \\
\text { equivocados durante a coleta do } \\
\text { exame; } \\
\text { - Conhecimento frágil tratado de } \\
\text { forma sucinta e com pouca } \\
\text { sustentação teórica. }\end{array}$ \\
\hline
\end{tabular}


O principal problema foi em relação ao período para coleta do TP. Esse exame é apenas um teste de triagem, ou seja, um resultado alterado não implica em diagnóstico definitivo de qualquer uma das doenças necessitando de exames confirmatórios. Portanto, é importante a realização do teste em tempo hábil, ou seja, na primeira semana de vida do neonato para que em casos positivos, se inicie o tratamento precocemente evitando sequelas como a deficiência intelectual e/ou até mesmo a morte. Pesquisas também têm mostrado uma baixa compreensão sobre TP pelas mães. Esse conhecimento superficial é referente à importância do teste, como ele é realizado, quais as doenças são detectadas, qual o período correto, entre outras. ${ }^{7,13-18}$ Algumas mães confundiram o TP com o "carimbo" do pé do bebê (impressão plantar) colhido logo após nascimento no Hospital/Maternidade e acreditaram já terem realizado o teste em seu filho. ${ }^{17} \mathrm{Um}$ desses estudos revelou que $97 \%$ das mães já tinham ouvido falar sobre o TP, mas não sabiam da sua finalidade e importância. Este resultado é preocupante pois as mães desconhecem um exame simples mas de extrema relevância para a saúde da criança. Os achados dessa pesquisa também evidenciaram falhas no acompanhamento pré-natal, nos postos de coleta e na maternidade, uma vez que não basta informar da necessidade de realização do teste, e sim, que se explique seus benefícios quando realizado precocemente. $^{13}$

Quanto às informações sobre o TP, uma pesquisa mostrou que apenas cinco mães (10\%) referiram receber orientação no pré-natal, embora $40 \quad(80 \%)$ delas tenham sido consultadas nesse período. Outros dados importantes foram: 1) $70 \%$ (35) das mulheres obtiveram informações sobre 0 teste ao receber alta da maternidade onde deram a luz; 2) 50\% (25) das mães entrevistadas levaram suas crianças a Unidade Básica de Saúde para realizar o TP entre o $7^{\circ}$ e $15^{\circ}$ dia de vida do RN e 3) $80 \%$ (40) delas não sabiam responder quais doenças o TP detecta. ${ }^{16}$ Outro estudo com 55 puérperas multíparas mostrou que algumas não realizaram o TP em seu filho por acharem o procedimento doloroso e 42,8\% (21) delas não souberam informar qual a idade recomendada para realização do teste. ${ }^{17}$ Esse último resultado foi confirmado por um estudo prévio, que avaliou a TN no Estado de Mato Grosso e mostrou que: 1) apenas $22 \%$ das amostras foram coletadas na idade recomendada e 2) a maioria realizou o teste de triagem entre 8 e 30 dias de vida do RN. Ainda, cerca de $10 \%$ das crianças teve amostras coletadas em idade superior a 30 dias. Assim, a idade na coleta e o atraso na fase de confirmação diagnóstica foram os principais motivos 
para o atraso do início do tratamento dos casos detectados pelo serviço. ${ }^{19}$

Os dados apresentados anteriormente enfatizam que o TP, apesar de sua extrema importância para a promoção da saúde do RN, parece ficar em segundo plano, tanto por parte dos profissionais de enfermagem quanto das mães, que enfocam suas preocupações principalmente com os cuidados com o neonato em relação à amamentação e higiene pessoal. $^{18}$ Deste modo, a desinformação ou incipiência de entendimento sobre TP de pais e profissionais de saúde pode influenciar negativamente na realização do teste, comprometendo o diagnóstico precoce e o início do tratamento. O conhecimento sobre os danos causados pelas doenças e a necessidade de tratamento imediato podem influenciar positivamente na realização do exame em período adequado e no interesse em buscar o resultado. ${ }^{17}$

Ainda, outros achados intrigantes referidos pela literatura são que as informações sobre a $\mathrm{TN}$ foi passada as mães após a alta hospitalar na maior parte dos $\operatorname{casos}^{7,14,17}$ e a orientação foi realizada por médicos. ${ }^{14}$ Apenas uma das 15 mães que realizaram o pré-natal, foi orientada sobre o TP durante esse período. ${ }^{14}$ Considerando que o enfermeiro é o profissional que mantém contato direto com a mãe desde o pré-natal até o puerpério e tem como atribuição a Educação em Saúde, essas orientações deveriam ser dadas por ele, profissional habilitado para isso, durante o ciclo gravídico-puerperal e não se restringir apenas ao pós-parto e ao momento da coleta do exame. Em suma, o exame do TP é uma medida preventiva que compete à Enfermagem, que tem fundamental participação no processo de implementação do PNTN, e deve esclarecer aos cuidadores sobre a importância e a finalidade do exame, do procedimento em si, bem como, da necessidade de buscar o resultado. ${ }^{11} \mathrm{O}$ entendimento limitado das mães sobre TN é, sem dúvida, reflexo das informações que receberam acerca do teste e destacam a fragilidade da atuação da Equipe de Enfermagem na Educação em Saúde.

Os resultados dos estudos apresentados acima $^{6,8,9-12}$ enfatizaram a necessidade de capacitação para os profissionais de Enfermagem, com instruções sobre coleta, importância, finalidade do teste, período de coleta, distúrbios triados, entre outras. $\mathrm{O}$ enfermeiro tem papel imprescindível na $\mathrm{TN}$, pois, deve promover a saúde e prevenir doenças. Portanto, é indispensável à implementação da Educação Permanente em Saúde visando efetiva qualificação desses profissionais. $\mathrm{O}$ enfermeiro qualificado para o exercício profissional 
desempenha suas funções de forma eficiente e eficaz, garantindo uma assistência global e humanizada. ${ }^{11}$

Os achados dos estudos prévios são preocupantes, nos fazem repensar a prática de Enfermagem na $\mathrm{TN}^{11}$ e nos remete as seguintes indagações: 1) será que os profissionais de Enfermagem possuem um conhecimento sólido para repasse dessas informações aos pais? 2) esses profissionais estão preparados para assistir e oferecer às famílias suporte integral à saúde do neonato em relação à TN? 3) como o tema TN e TP é abordado nas Cursos de Graduação em Enfermagem no Brasil? 4) não seria necessário a promoção continuada de cursos de capacitação a esses profissionais para que os mesmos possam ter segurança na orientação e execução do exame? Em resposta a essa última questão, um estudo recente mostrou mudança significativa de conhecimento dos profissionais de Enfermagem após ações educativas em Triagem Auditiva Neonatal, na maioria das variáveis analisadas. ${ }^{20}$ Outro profissional de saúde que ocupa um lugar proeminente na TN é o pediatra, no entanto, o seu conhecimento das doenças triadas, do tratamento e do prognóstico também foi limitado. ${ }^{3}$

\section{CONSIDERAÇÕES FINAIS}

Esse trabalho tem por finalidade refletir e re(pensar) a atuação da Enfermagem no TP. Esse profissional deve ter um amplo conhecimento sobre a TN, visando à questão da orientação das mães, tendo em vista sua interação direta com a clientela alvo: mãe (gestante e/ou puérpera) e RN. Essa reflexão mais profunda sobre a temática se faz necessário tendo que em vista que a Enfermagem é uma profissão que pode contribuir para a efetividade deste programa, favorecendo a elevação da cobertura e número de exames. No entanto, para que a Enfermagem atue na promoção da saúde e prevenção de doenças, é indispensável que o profissional reflita sobre seu papel social e busque continuamente por capacitação.

Em síntese, o TP é um tema de importância inquestionável, pois, permite o diagnóstico precoce de doenças genéticas, assintomáticas no período neonatal e possibilita o tratamento precoce específico. Os dados apresentados acima mostraram que as mães/pais não possuem o conhecimento da relevância do exame para o futuro de seus filhos. Acrescente-se a esse quadro a constatação de que os profissionais de enfermagem parecem desinformados quanto a TN e necessitam de capacitação para melhor propagação e execução de ações educativo-preventiva em torno dessa temática. O estímulo à educação continuada em saúde para esses profissionais lhes proporcionará uma melhoria na qualidade da assistência prestada. 


\section{REFERÊNCIAS}

1. Ministério da Saúde. Manual de normas técnicas e rotinas operacionais do programa nacional de triagem neonatal. 2. ed. ampl. Brasília (DF): Ministério da Saúde; 2004.

2. Sociedade Brasileira de Triagem Neonatal. [página na Internet; acesso em 2015 Jan 28]. Disponível em: http://www.sbtn.org.br

3. Leão LL, Aguiar MJB. Triagem neonatal: o que os pediatras deveriam saber. J Pediatr (Rio J) 2008; 84(4):S8090.

4. Ministério da Saúde. Portaria GM/MS n ${ }^{\circ}$ 822, de 06 de junho de 2001: institui o Programa Nacional de Triagem Neonatal (PNTN). Brasília (DF): Ministério da Saúde; 2001.

5. Ministério da Saúde. Portaria no. 2.829, de 14 de dezembro de 2012. Inclui a fase IV no Programa Nacional de Triagem Neonatal (PNTN). Brasília (DF): Ministério da Saúde; 2012.

6. Silva MBGM, Zagonel IS, Lacerda MR. Enfermagem na triagem neonatal. Acta Scientiarum Health Sciences 2003; 25(2):155-61.

7. Abreu IS, Braguini WL. Triagem neonatal: o conhecimento materno em uma maternidade no interior do Paraná, Brasil. Rev Gaúcha Enferm 2011; 32(3):596-601. 8. Silva MBGM, Zagonel IS, Lacerda MR. Cuidados de Enfermagem e o Teste do Pezinho. Cogitare Enferm. 2002; 7(1):437.

9. Pimente EDC, Luz GS, Schiavon GB, Pelloso SM, Carvalho MDB. Teste do Pezinho: A Humanização do Cuidado e do Profissional. REME-Rev. Min. Enferm. 2010; 14(1):25-8.

10. Benincasa TO, Oliveira CB, Zanoni IH, Lima SAO, Martins DC. Triagem Neonatal: a percepção teórica da equipe de enfermagem da Unidade de Terapia Intensiva Neonatal. Rev Inst Ciênc Saúde 2009; 27(2):109-14.

11. Acosta DF, Strefling ISS, Gomes VLO. Triagem Neonatal: (Re)Pensando a Prática de Enfermagem. Rev Enferm UFPE online 2013; 7(2):572-8.

12. Strefling ISS, Monfrim XM, Lunardi Filho WD, Carvalho KK, Azevedo ALS. Conhecimento sobre Triagem Neonatal e sua Operalização. Cogitare Enferm. 2014; 19(1):27-33.

13. Reichert APS, Pacífico VC. Conhecimento de mães quanto a importância do Teste do Pezinho. Rev Bras Enferm. 2003; 56(3):226-9.

14. Amorim JF, Souza MHN. O conhecimento das mães acerca da triagem neonatal. R Enferm UERJ 2005; 13(1):2731.

15. Garcia MG, Ferreira EAP, Oliveira FPS. Análise da compreensão de pais acerca do Teste do Pezinho. Rev Bras Crescimento Desenvolv Hum 2007; 17(1):1-12.

16. Salles M, Santos IMM. O conhecimento das mães acerca do teste do pezinho em uma unidade básica de saúde. Rev. Pesq: Cuidado é Fundamental On line. 2009; 1(1):59-64

17. Santos EC, Gaíva MAM, Santos JG, Abud SM. O conhecimento de puérperas sobre a triagem neonatal. Cogitare Enferm. 2011; 16(2):282-8.

18. Al-Alam AC, Soares MC, Meincke SMK, Dilélio AS, Escobal APL. Entendimento das Mães acerca da triagem neonatal: um estudo qualitativo. J Nurs Health 2012; 1(2):75-81.

19. Stranieri I, Takano OA. Avaliação do Serviço de Referência em Triagem Neonatal para hipotireoidismo congênito e fenilcetonúria no Estado de Mato Grosso, Brasil. Arq Bras Endocrinol Metab 2009; 53(4):446-52.

20. Barbosa CP, Aires JB, Farias IYS, Linhares FMP, Griz SMS. Educação em saúde auditiva do neonato e lactente para profissionais de enfermagem. Braz $\mathbf{J}$ Otorhinolaryngol 2013; 79(2):226-32.

Recebido em 13/03/2016

Aprovado em 29/08/2016

Publicado em 29/12/2016 